\title{
The Order of Accuracy of Quadrature Formulae for Periodic Functions
}

\author{
Alexander Kurganov and Jeffrey Rauch \\ We dedicate this paper to Feruccio Colombini on the event of his sixtieth birthday. He has been \\ an inspiring colleague, coauthor and close friend. We wish him happy and creative years till \\ the next milestone.
}

\begin{abstract}
The trapezoidal quadrature rule on a uniform grid has spectral accuracy when integrating $C^{\infty}$ periodic function over a period. The same holds for quadrature formulae based on piecewise polynomial interpolations. In this paper, we prove that these quadratures applied to $W_{\text {per }}^{r, p}$ periodic functions with $r>2$ and $p \geq 1$ have error $\mathcal{O}\left((\Delta x)^{r}\right)$. The order is independent of $p$, sharp, and for $p<\infty$ is higher than predicted by best trigonometric approximation. For $p=1$ it is higher by 1 .
\end{abstract}

Mathematics Subject Classification (2000). Primary 65D32; Secondary 65T40.

Keywords. Numerical quadrature, periodic function, order of accuracy, trigonometric polynomial, piecewise polynomial interpolation, trapezoidal rule.

\section{Upper bound on the error}

Denote by $W_{\text {per }}^{r, p}$ the Banach space of periodic functions on $\mathbb{R}$ whose distribution derivatives up to order $r$ belong to $L_{\text {per }}^{p}(\mathbb{R})$. The norm is equal to the sum of the $L^{p}$ norms of these derivatives over one period. Without loss of generality we take the period equal to $2 \pi$. Introduce a partition of the interval $[0,2 \pi]$ into $N$ equal subintervals of size $\Delta x:=2 \pi / N$.

The research of A. Kurganov was supported in part by the National Science Foundation under grants DMS-0310585 and DMS-0610430. The research of J. Rauch was supported in part by the National Science Foundation under grant DMS-0405899. 
Because of the periodicity, the trapezoidal rule is,

$$
\int_{0}^{2 \pi} f(x) d x \approx T_{N}(f):=\Delta x \sum_{j=0}^{N-1} f\left(x_{j}\right), \quad x_{j}:=j \Delta x, \quad j=0,1, \ldots, N-1 .
$$

The error is equal to

$$
E_{N}(f):=T_{N}(f)-\int_{0}^{2 \pi} f(x) d x
$$

Write $f$ as the sum of its Fourier series,

$$
f(x)=\sum_{n \in \mathbb{Z}} c_{n} e^{i n x}, \quad c_{n}:=\frac{1}{2 \pi} \int_{0}^{2 \pi} f(x) e^{-i n x} d x .
$$

Denote by $\mathcal{P}(m)$ the set of all trigonometric polynomials of degree at most $m$, that is functions of the form

$$
\sum_{n=-m}^{m} a_{n} e^{i n x}, \quad a_{n} \in \mathbb{C}
$$

Summing finite geometric series shows that $T_{N}\left(e^{i n x}\right)=0$ for $0<|n|<N$ and it follows that $T_{N}$ exactly integrates trigonometric polynomials of degree $N-1$. Therefore, for any $P \in \mathcal{P}(N-1)$,

$$
E_{N}(f)=E_{N}(f-P)=T_{N}(f-P)-\int_{0}^{2 \pi}(f(x)-P(x)) d x .
$$

This yields the bound in terms of best trigonometric approximation

$$
\left|E_{N}(f)\right| \leq 4 \pi \inf _{P \in \mathcal{P}(N-1)}\|f-P\|_{L^{\infty}} .
$$

Spectral accuracy then follows for infinitely smooth $f$ thanks to the rapid decay of the Fourier coefficients.

Our estimate proceeds differently. For any integer $k$ the functions $e^{i n x}$ and $e^{i(n+k N) x}$ agree at the nodes for $T_{N}$. Therefore, $T_{N}\left(e^{i n x}\right)=T_{N}\left(e^{i(n+k N) x}\right)$ and thus, $T_{N}\left(e^{i n x}\right)=0$ for all $n \neq k N$ and $T_{N}\left(e^{i n x}\right)=2 \pi$ for all $n=k N$. This can also be checked by summing the corresponding finite geometric series. It follows that

$$
E_{N}(f)=2 \pi \sum_{0 \neq k \in \mathbb{Z}} c_{k N}=2 \pi \sum_{0 \neq k \in \mathbb{Z}}\left((i k N)^{r} c_{k N}\right) \frac{1}{(i k N)^{r}} .
$$

This involves only a small fraction of the Fourier coefficients $c_{n}$ with $|n| \geq N$. 
Theorem 1.1. If $f \in W_{\mathrm{per}}^{r, 1}$ and $1<r \in \mathbb{N}$, then the error of the trapezoidal quadrature rule (1.1) satisfies

$$
\left|E_{N}(f)\right| \leq \frac{C\left\|f^{(r)}\right\|_{L^{1}([0,2 \pi])}}{N^{r}}, \quad f^{(r)}:=\frac{d^{r} f}{d x^{r}}, \quad C:=2 \sum_{k=1}^{\infty} \frac{1}{k^{r}} .
$$

Remark 1.2. The result is interesting only for $r>2$, since for $1 \leq r \leq 2$ the $N^{-r}$ convergence rate can be established using standard arguments even in the case of nonperiodic $f$.

Remark 1.3. Analogous estimates are true for noninteger $r$. For $r \geq 2$ they can be obtained by interpolation between integer values.

Proof. Introduce

$$
Q_{N}(x):=\frac{1}{N} \sum_{j=0}^{N-1} \delta\left(x-x_{j}\right), \quad g_{N}(x):=\sum_{0 \neq k \in \mathbb{Z}} \frac{1}{(i k N)^{r}} e^{i k N x},
$$

where $\delta$ is the Dirac delta-function. The $n^{\text {th }}$ Fourier coefficient of $Q_{N}$ is equal to $T_{N}\left(e^{-i n x}\right) /(2 \pi)^{2}$ so

$$
Q_{N}(x)=\frac{1}{2 \pi} \sum_{k \in \mathbb{Z}} e^{i k N x}
$$

This together with $f^{(r)}(x)=\sum_{n \in \mathbb{Z}}(i n)^{r} c_{n} e^{i n x}$ yields

$$
\sum_{0 \neq k \in \mathbb{Z}}(i k N)^{r} c_{k N} e^{i k N x}=Q_{N} * f^{(r)}(x),
$$

where $*$ denotes convolution of periodic functions. Equation (1.3) then implies

$$
E_{N}(f)=\int_{0}^{2 \pi} Q_{N} * f^{(r)}(x) g_{N}(-x) d x .
$$

Since $Q_{N}$ is a measure with total variation per period equal to 1 , one has,

$$
\left\|\sum_{0 \neq k \in \mathbb{Z}}(i k N)^{r} c_{k N} e^{i k N x}\right\|_{L^{1}([0,2 \pi])}=\left\|Q_{N} * f^{(r)}\right\|_{L^{1}([0,2 \pi])} \leq\left\|f^{(r)}\right\|_{L^{1}([0,2 \pi])} .
$$

On the other hand,

$$
\left\|g_{N}\right\|_{L^{\infty}} \leq \sum_{0 \neq k \in \mathbb{Z}} \frac{1}{|k N|^{r}}=\frac{2}{N^{r}} \sum_{k=1}^{\infty} \frac{1}{k^{r}}
$$

and the sum is finite for $r>1$. Combining (1.5), (1.6) and (1.7) yields (1.4). 
Remark 1.4. The order of accuracy established in Theorem 1.1 for the trapezoidal rule, is also true for other quadratures based on piecewise polynomial interpolations. This is so since such quadratures applied to periodic functions can be rewritten as a convex combination of trapezoidal rules with shifted nodes (see [2]).

\section{A lower bound and comparison with best trigonometric approximation}

For integer $r>1, W_{\text {per }}^{r, p}$ consists of $C^{r-1}$ functions so that $f^{(r-1)}$ is absolutely continuous with derivative in $L_{\mathrm{per}}^{p}$. For $p>1, f^{(r-1)}$ belongs to the Hölder class $C^{r-1, \alpha}$ with $\alpha=1-1 / p$. The right hand side of (1.2) is largely predicted by the modulus of continuity of $f^{(r-1)}$ (see $[1,3]$ ). The rate of best approximation is different for the different spaces $W_{\text {per }}^{r, p}$ with $r>1$ fixed and $1 \leq p \leq \infty$. In contrast, the order of convergence of the trapezoidal rule is essentially independent of $p$ as the following example shows.

Example. Define $f$ by the lacunary Fourier series:

$$
f(x):=\sum_{n=1}^{\infty} \frac{1}{\left(2^{n}\right)^{r}} e^{i 2^{n} x} .
$$

Then $\int_{0}^{2 \pi} f(x) d x=0$. In addition, $f \in W_{\mathrm{per}}^{r-\varepsilon, \infty}$ for all $\varepsilon>0$ and the error in the trapezoidal approximation $T_{2^{N}}(f)$ is exactly equal to

$$
E_{2^{N}}(f)=2 \pi \sum_{2^{n} \text { is a multiple of } 2^{N}} \frac{1}{\left(2^{n}\right)^{r}}=2 \pi \sum_{k=0}^{\infty} \frac{1}{\left(2^{N+k}\right)^{r}}=\frac{1}{\left(2^{N}\right)^{r}} \frac{2^{r+1} \pi}{2^{r}-1} .
$$

As this is $\mathcal{O}\left(\left(2^{N}\right)^{-r}\right)$, the rate of convergence for $W_{\text {per }}^{r, \infty}$ cannot be better, in the sense of a higher power of $1 / N$, than that for $W_{\text {per }}^{r, 1}$.

\section{Conclusion}

The trapezoidal rule and other quadrature formulae based on piecewise polynomial interpolations have error $\mathcal{O}\left((\Delta x)^{r}\right)$ for functions in $W_{\text {per }}^{r, p}$. The rate is independent of $p$ and is optimal in the sense that no higher power of $\Delta x$ is possible. The error is that which is predicted by approximation theory for functions in $W_{\text {per }}^{r, \infty}$ and it is interesting that it remains true for the elements of $W_{\text {per }}^{r, 1}$, for which the best approximation by trigonometric polynomials of degree $N-1$ is not as small as $\mathcal{O}\left((\Delta x)^{r-1+\varepsilon}\right)$. For $W_{\text {per }}^{r, 1}$, the rate of convergence is essentially a full order more rapid than that given by best approximation as in (1.2). 


\title{
References
}

[1] J. Favard, Sur les meilleurs procédés d'approximation de certaines classesde fonctions par des polynômes trigonométriques, Bull. Sci. Math. 61 (1937), 209-224, 243-256.

[2] A. Kurganov and S. Tsynkov, On spectral accuracy of quadrature formulae based on piecewise polynomial interpolations, Center for Research in Scientific Computation, North Carolina State University, Technical Report No. CRSC-TR07-11, 2007; available at http://www.ncsu.edu/crsc/reports/ftp/pdf/crsc-tr07-11.pdf.

[3] A.F. Timan, Theory of approximation of functions of a real variable. Translated from Russian by J. Berry. English translation edited and editorial preface by J. Cossar. International Series of Monographs in Pure and Applied Mathematics, A Pergamon Press Book. The Macmillan Co., New York, 1963.

\section{Acknowledgment}

Most of this work was done in Fall 2005, when A. Kurganov visited the Department of Mathematics of the University of Michigan during the semester following hurricane Katrina. A. Kurganov thanks the chairman Professor A. Bloch and all members of the department for their extremely warm hospitality.

\author{
Alexander Kurganov \\ Mathematics Department \\ Tulane University \\ New Orleans, LA 70118 \\ USA \\ e-mail: kurganov@math.tulane.edu \\ Jeffrey Rauch \\ Department of Mathematics \\ University of Michigan \\ Ann Arbor, MI 48109 \\ USA \\ e-mail: rauch@umich.edu
}

